\title{
EVALUATION DE LA QUALITÉ DES PRODUITS DU CANARD GRAS *
}

\author{
EVALUATION OF THE QUALITY OF FAT DUCK PRODUCTS
}

Evaluación de la CAlidAd de PROductos de PATO

\author{
D. Bastianelli ${ }^{1 *}$ L. Bonnal $^{1}$
}

\section{Mots-clés : Canard - Qualité des aliments - Spectrométrie dans le proche infrarouge.}

Keywords: Duck - Food quality - Near infrared spectroscopy.

Palabras clave: Pato - Calidad de los alimentos -

Espectroscopía de infrarrojo cercano.

L: a caractérisation des produits animaux est importante autant - pour le suivi de leur qualité que pour la recherche de leur amélioration par des voies d'amélioration génétique ou de procédés technologiques. L'enjeu est particulièrement important pour des filières à forte valeur ajoutée, comme le canard gras.

Dans le cadre d'essais sur l'amélioration génétique du canard (2), des méthodes rapides - spectroscopie proche infrarouge (SPIR) - de prédiction de la qualité ont été testées pour le foie gras et pour le filet (magret). Il s'agissait de permettre la mesure sur un grand nombre d'échantillons de paramètres dont la méthode de référence est particulièrement longue (matières grasses [MG] par extraction Folch, rendement technologique, entre autres) et d'étudier la possibilité de les appliquer ensuite dans I'industrie.

Les spectres des échantillons ont été acquis sur un spectromètre ASD Labspec, directement sur le produit entier (foie ou magret), sans préparation. L'étalonnage a été réalisé avec des mesures de composition chimique (matière sèche [MS], minéraux, protéines, MG) ou technologique (par exemple taux de fonte pour le foie, pertes à la cuisson pour le filet) (1).

La précision des étalonnages a varié selon les paramètres (tableau I). Les paramètres de composition proximale ont globalement été bien prédits (MS, MG, protéines) avec des valeurs de $R^{2}$ comprises entre 0,80 et 0,90 . Certains constituants biochimiques comme le collagène ont été moins facilement prédits $\left(R^{2}=0,19\right)$, soit par manque de variabilité dans la population, soit par des défauts dans les mesures de référence. Les propriétés technologiques du filet sont difficiles à approcher par SPIR, avec par exemple une valeur de $\mathrm{R}^{2}=0,19$ pour la prédiction de la perte à la cuisson. En revanche, la qualité technologique du foie

1. Cirad, UMR Selmet, TAC112/A, avenue Agropolis, 34398 Montpellier Cedex 5, France.

* Auteur pour la correspondance

E-mail : denis.bastianelli@cirad.fr

$\$$ Ce texte est issu d'une communication orale présentée aux journées scientifiques QualiREG 2014. L'ensemble des résultats a ensuite fait l'objet d'une publication scientifique (Marie-Etancelin et coll., 2014).

\section{Tableau I}

Précision des étalonnages obtenus pour la qualité du foie et du magret

$\begin{array}{lccc} & \begin{array}{c}\text { Moyenne } \\ (\mathbf{\%})\end{array} & \begin{array}{c}\text { Erreur de mesure } \\ (\text { SECV) }\end{array} & \mathbf{R}^{\mathbf{2}} \\ \text { Foie } & & & \\ \text { Matières grasses } & 51,0 & 2,24 & 0,89 \\ \text { Protéines } & 8,1 & 0,72 & 0,79 \\ \text { Collagène } & 1,5 & 0,24 & 0,19 \\ \text { Taux de fonte } & 35,8 & 6,50 & 0,85 \\ & & & 0,81 \\ \text { Magret } & 4,9 & 0,53 & 0,25 \\ \text { Matières grasses } & 22,2 & 3,10 & \\ \text { Perte cuisson } & & & \end{array}$

SECV : erreur standard de validation croisée

évaluée par le taux de fonte est assez bien prédite $\left(R^{2}=0,85\right)$ pour permettre une évaluation objective du produit.

Dans un second temps les étalonnages réalisés ont été appliqués à l'étude de la variabilité intra-échantillon : 46 spectres acquis sur toute la surface d'un foie gras ont permis d'établir des cartes de répartition des constituants et des propriétés des foies, comme le montre la figure 1 pour le taux de fonte.

Les calibrations réalisées ont permis de prédire la composition chimique de plusieurs milliers d'échantillons, et d'évaluer les paramètres génétiques (héritabilité) et de rechercher des locus de caractères quantitatifs (2). La base de données générée a également permis de décrire la variabilité des paramètres de qualité. L'étude de la variabilité de composition au sein même d'un échantillon permet de mieux comprendre l'élaboration des propriétés technologiques. Par ailleurs des essais sont actuellement en cours chez un industriel pour utiliser ces résultats dans la caractérisation du foie en routine.

\section{BIBLIOGRAPHIE}

1. BASTIANELLI D., BRACHET M., BONNAL L., 2013. Comment un étalonnage global peut-il décrire I'hétérogénéité intra-échantillon? Exemples sur le foie gras. In : $14^{\mathrm{es}}$ Rencontres Hélio-SPIR, Spectrométrie proche infrarouge et hétérogénéité, Montpellier, France, 13 nov. 2013.

2. MARIE-ETANCELIN C., VITEZICA Z.G., BONNAL L., FERNANDEZ X., BASTIANELLI D., 2014. Selecting the quality of mule duck fatty liver based on near-infrared spectroscopy. Gen. Sel. Evol., 46: 38.

Accepted 30 April 2015; Online publication June 2015 
Proceedings of QualiREG 2012-2014 Scientific Days

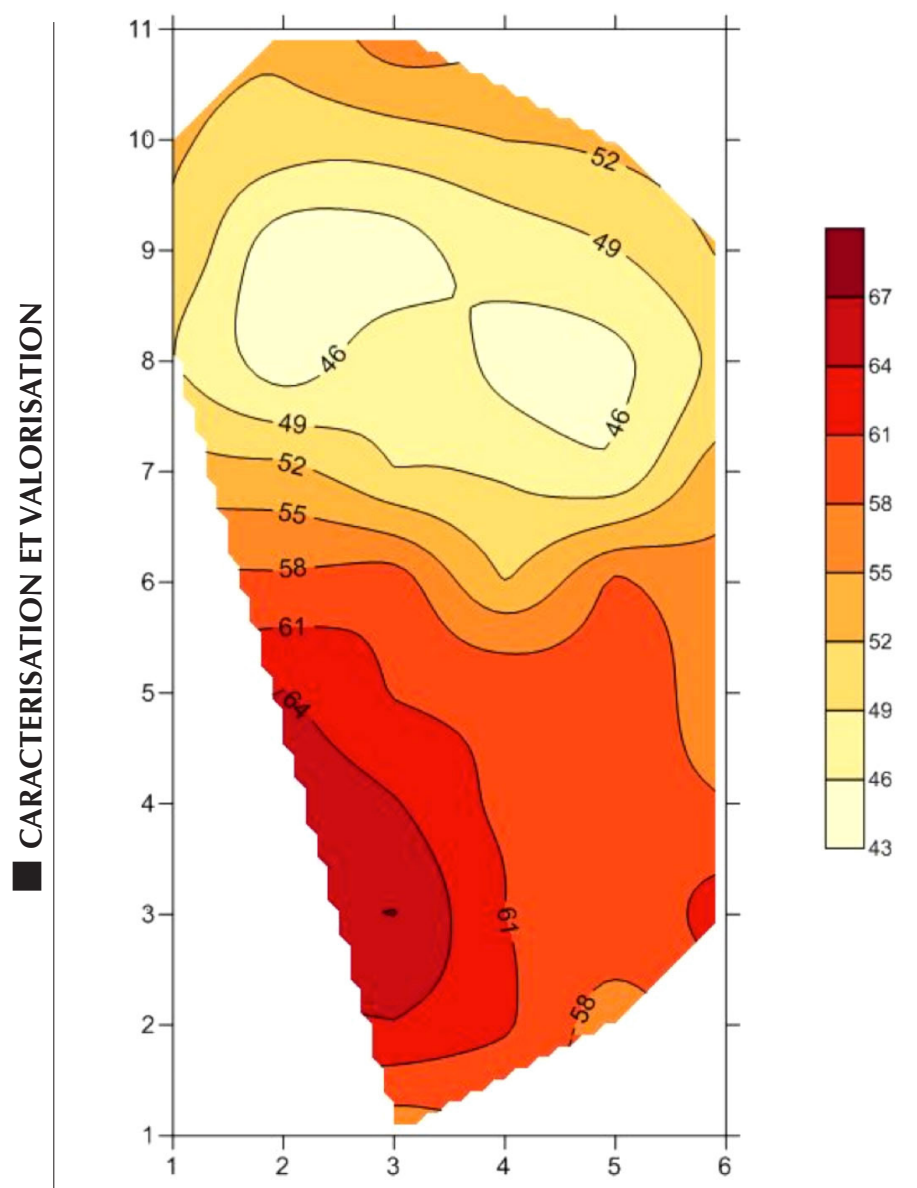

Figure 1 : répartition du taux de fonte au sein d'un échantillon de foie gras. 